OPEN ACCESS

Edited by:

Joel S. Greenberger,

University of Pittsburgh Medical

Center, USA

Reviewed by:

Wenyin Shi,

Thomas Jefferson University, USA

Patrik Brodin,

Albert Einstein College of

Medicine, USA

*Correspondence:

Ralph S. DaCosta

rdacosta@uhnres.utoronto.ca

${ }^{\dagger}$ Marieke A. Stammes and Azusa Maeda contributed equally to this work.

Specialty section: This article was submitted to

Radiation Oncology,

a section of the journa

Frontiers in Oncology

Received: 19 July 2016 Accepted: 05 October 2016 Published: 21 October 2016

Citation:

Stammes MA, Maeda A, Bu J,

Scollard DA, Kulbatski I,

Medeiros PJ, Sinisi R,

Dubikovskaya EA, Snoeks TJA,

van Beek ER, Chan $A B$,

Löwik CWGM and DaCosta RS

(2016) The Necrosis-Avid Small

Molecule HQ4-DTPA as a

Multimodal Imaging Agent for

Monitoring Radiation Therapy-

Induced Tumor Cell Death.

Front. Oncol. 6:221.

doi: 10.3389/fonc. 2016.00221

\section{The Necrosis-Avid Small Molecule HQ4-DTPA as a Multimodal Imaging Agent for Monitoring Radiation Therapy-Induced Tumor Cell Death}

\author{
Marieke A. Stammes ${ }^{1,2 t}$, Azusa Maeda ${ }^{3,4+}$, Jiachuan Bu ${ }^{3}$, Deborah A. Scollard, \\ Iris Kulbatski ${ }^{3}$, Philip J. Medeiros ${ }^{3}$, Riccardo Sinisi', Elena A. Dubikovskaya ${ }^{6}$, \\ Thomas J. A. Snoeks ${ }^{1}$, Ermond R. van Beek', Alan B. Chan², Clemens W. G. M. Löwik ${ }^{7}$ and \\ Ralph S. DaCosta ${ }^{3,4,8 *}$

\begin{abstract}
${ }^{1}$ Department of Radiology, Leiden University Medical Center, Leiden, Netherlands, ${ }^{2}$ Percuros BV, Enschede, Netherlands, ${ }^{3}$ Princess Margaret Cancer Center, University Health Network, Toronto, ON. Canada, ${ }^{4}$ Department of Medical Biophysics, University of Toronto, Toronto, ON, Canada, ${ }^{5}$ STTARR Innovation Centre, University Health Network, Toronto, ON, Canada, ${ }^{6}$ LCBIM, Institute of Chemical Sciences and Engineering, Swiss Federal Institute of Technology of Lausanne (EPFL), Lausanne, Switzerland, ${ }^{7}$ Department of Radiology, Erasmus Medical Center, Rotterdam, Netherlands, ${ }^{8}$ Techna Institute, University Health Network, Toronto, ON, Canada
\end{abstract}

Purpose: Most effective antitumor therapies induce tumor cell death. Non-invasive, rapid and accurate quantitative imaging of cell death is essential for monitoring early response to antitumor therapies. To facilitate this, we previously developed a biocompatible necrosis-avid near-infrared fluorescence (NIRF) imaging probe, HQ4, which was radiolabeled with ${ }^{111}$ Indium-chloride $\left({ }^{111} \mathrm{In}-\mathrm{Cl}_{3}\right)$ via the chelate diethylene triamine pentaacetic acid (DTPA), to enable clinical translation. The aim of the present study was to evaluate the application of HQ4-DTPA for monitoring tumor cell death induced by radiation therapy. Apart from its NIRF and radioactive properties, HQ4-DTPA was also tested as a photoacoustic imaging probe to evaluate its performance as a multimodal contrast agent for superficial and deep tissue imaging.

Materials and methods: Radiation-induced tumor cell death was examined in a xenograft mouse model of human breast cancer (MCF-7). Tumors were irradiated with three fractions of 9 Gy each. HQ4-DTPA was injected intravenously after the last irradiation, NIRF and photoacoustic imaging of the tumors were performed at 12, 20, and $40 \mathrm{~h}$ after injection. Changes in probe accumulation in the tumors were measured in vivo, and ex vivo histological analysis of excised tumors was performed at experimental endpoints. In addition, biodistribution of radiolabeled [ ${ }^{111}$ In]DTPA-HQ4 was assessed using hybrid single-photon emission computed tomography-computed tomography (SPECT-CT) at the same time points.

Results: In vivo NIRF imaging demonstrated a significant difference in probe accumulation between control and irradiated tumors at all time points after injection. A similar trend was observed using in vivo photoacoustic imaging, which was validated by ex vivo tissue fluorescence and photoacoustic imaging. Serial quantitative radioactivity measurements of probe biodistribution further demonstrated increased probe accumulation in irradiated tumors. 
Conclusion: HQ4-DTPA has high specificity for dead cells in vivo, potentiating its use as a contrast agent for determining the relative level of tumor cell death following radiation therapy using NIRF, photoacoustic imaging and SPECT in vivo. Initial preclinical results are promising and indicate the need for further evaluation in larger cohorts. If successful, such studies may help develop a new multimodal method for non-invasive and dynamic deep tissue imaging of treatment-induced cell death to quantitatively assess therapeutic response in patients.

Keywords: radiation therapy, cell death, multimodal imaging, fluorescence imaging, cyanine, necrosis avid contrast agent, treatment response, cancer

\section{INTRODUCTION}

The International Agency for Research on Cancer estimated that globally in 2012, 14.1 million new patients were diagnosed with cancer and that this number will increase to more than 20 million in 2025 (1). After diagnosis, most patients with solid tumors undergo surgery, radiotherapy and/or chemotherapy, and may be followed up with alternative treatments. Conventional methods for monitoring antitumor treatment response are based on anatomical imaging, e.g., X-ray, magnetic resonance imaging (MRI) and computed tomography (CT) every 6-8 weeks during the course of treatment as described in the Response Evaluation Criteria In Solid Tumors (RECIST) (2). Although RECIST provides a standardized guideline, assessment of treatment efficacy based on gross tumor size alone may be insufficient for certain organs and treatments (3). Moreover, volumetric change in tumor size based on conventional imaging may be a delayed indicator of treatment effectiveness (4), unnecessarily exposing patients to the side effects of additional ineffective treatments, and postponing treatment adjustment. Thus, there is a need for novel imaging methods to assess tumor response early and at a cellular/molecular level in order to determine treatment efficacy accurately and adjust the therapy based on tumor response $(5,6)$. Ideally, such methods would be non-invasive, clinically practical, and have sufficient sensitivity and specificity for tumor cell death in real time.

Imaging of treatment-induced tumor necrosis may facilitate quantitation of early treatment response in solid tumors as an alternative to the conventional radiological volumetric imaging. First, antitumor therapies, such as radiation therapy are known to induce several forms of tumor cell death that will often lead to secondary necrosis (7-9). Second, necrosis is primarily induced by external factors that cause physiochemical damage compared to apoptosis that can occur in any tissues during normal development and cell turnover $(10,11)$, making necrosis-based imaging method suitable to distinguish cell death induced by antitumor therapies. Finally, tumor necrosis, secondary to ischemia and insufficient vascularization to support a rapidly proliferating tumor mass (12), has been positively correlated with the aggressiveness of cancer, and, therefore, has been used as a diagnostic biomarker for cancer staging (13-18). Thus, exogenous imaging contrast agents that specifically bind to necrotic tumor cells in vivo could enable accurate determination of treatment effects and disease staging, as well as earlier prediction of treatment outcomes for solid tumors (19).

Accurate quantification of tissue necrosis may have wide clinical relevance compared to conventional practice, especially in monitoring the efficacy of antitumor therapies at earlier stages. Existing necrosis-based imaging agents can be divided into two general groups: (1) MRI and CT contrast agents that enhance endogenous tissue necrosis contrast non-specifically by enabling visualization of the presence of an avascular necrotic core and (2) positron emission tomography (PET) and single-photon emission computed tomography (SPECT) contrast agents that are specifically targeted to endogenous necrotic tissue $(8,13-18$, 20-29). Non-specific tissue necrosis-imaging agents will likely fall into abeyance when affordable necrosis-specific agents become clinically available. Thus far, only a few agents have been considered clinically applicable, including necrosis-avid photosensitizer hypericin (Oncocidia $\left.{ }^{\mathrm{TM}}\right)(30-32)$ and ${ }^{131}$ Iodineconjugated Tumor Necrosis Targeting monoclonal antibody (TNT-3, Peregrine Pharmaceuticals, CA, USA) $(24,33)$. While clinical feasibility has been shown for both agents (34), several drawbacks may hinder their wide-spread clinical adoption (27, $30,35-37)$. For example, hypericin is phototoxic, poorly soluble, and aggregates rapidly. Monoclonal antibodies are relatively large in size, have long circulation times, may induce host immune response, and are expensive to develop using good manufacturing practices (GMP) (35-38).

Recognizing the biological significance of tumor necrosis as a hallmark of tumor response to treatment and the need for alternative imaging methods to measure treatment-induced solid tumor necrosis, we previously developed a biocompatible near-infrared fluorescence (NIRF), water-soluble imaging probe called HQ4. HQ4 is economical to produce, is non-phototoxic, and binds specifically to cells with compromised cell membrane integrity (38). We validated HQ4-diethylene triamine pentaacetic acid (HQ4-DTPA) as a necrosis-avid contrast agent histologically by demonstrating localization of HQ4-DTPA in necrotic tumors, and indicated that HQ4-DTPA could be made more clinically practical by addition of a radioactive moiety (38). Building on these results, in the current study, we investigated the utility of HQ4-DTPA as a necrosis-imaging agent in vivo to measure tumor response to radiation therapy. Since radiotherapy is used to treat over $50 \%$ of cancer patients (39), the translational value of HQ4DTPA is promising. 
In this study, we examined a relatively high-dose-per-fraction treatment scheme $(3 \times 9$ Gy) to induce tumor cell death. This regime was calculated based on a biological equivalent dose (BED) that is clinically relevant to 60 Gy for 2 Gy fractions, given a known $\alpha / \beta$ ratio of MCF-7 cells, comparable to the average $\alpha / \beta$ ratio of humans, and the incomplete repair model. We investigated a trimodal HQ4-DTPA imaging (photoacoustic, NIRF, SPECT) approach to measure tumor response to radiation therapy in a MCF-7 human breast cancer mouse xenograft model. We reasoned that the addition of photoacoustic imaging would overcome some of the disadvantages associated with SPECT and NIRF, such as the exposure to ionizing radiation emitted from radionuclides and the limited penetration depth (40), respectively. Photoacoustic imaging may also be ideal for routine clinical use as it is easily accessible, minimally invasive, and technologically inexpensive compared to conventional imaging methods (CT, MRI). The results of this work demonstrate the feasibility of using the multimodal (NIRF, photoacoustic, SPECT) HQ4-DTPA probe in vivo for longitudinal measurement of solid tumor necrosis in response to clinically relevant high-dose radiotherapy.

\section{MATERIALS AND METHODS}

\section{HQ Preparation}

HQ4-DTPA was obtained from Ilumicare BV (Rotterdam, The Netherlands). HQ4-DTPA was synthesized as previously described (38). For phantom studies, dilutions of HQ4DTPA were prepared in phosphate buffered saline (PBS) at various concentrations $(12.5,25,50$, and $100 \mu \mathrm{M})$. For in vivo mouse studies, $100 \mu \mathrm{L}$ that represents $10 \mathrm{nmol} \mathrm{HQ} 4$ DTPA was injected via the tail vein. To label HQ4-DTPA with ${ }^{111} \mathrm{InCl}_{3}$, HQ4-DTPA was dissolved in 0.1 M HEPES $(10 \mu \mathrm{g} / 100 \mu \mathrm{L})(41)$ and incubated with ${ }^{111} \mathrm{InCl}_{3}(35 \mathrm{MBq}$; Nordion, Vancouver, BC, USA). After $30 \mathrm{~min}$ of incubation on a shaker, labeling was validated with instant thin layer chromatography (ITLC). In all cases, labeling efficacy was greater than $90 \%$.

\section{Cell Culture}

GFP-fluorescent MCF-7 human breast cancer cells (kindly provided by Dr. Shirley Wu, Leslie Dan Faculty of Pharmacy, University of Toronto) were grown in DMEM Medium supplemented with $10 \%$ fetal bovine serum and $1 \%$ Pen-Strep in a humidified incubator at $37^{\circ} \mathrm{C}$ and $5 \% \mathrm{CO}_{2}$. Cells were trypsinized, counted, and suspended in 10\% PBS before further use.

\section{Animal Studies}

All animal procedures were conducted in accordance with appropriate regulatory standards under protocols AUP\#2407 and \#3004 approved by the University Health Network Institutional Animal Care Committee, and conform to the institutional guidelines for the proper and humane use of animals in research. Eight to 10-week-old female athymic nude mice (NCRNU-F strain) were obtained from Taconic Biosciences (Hudson, NY, USA). Approximately $2 \times 10^{6} \mathrm{MCF}-7-\mathrm{GFP}$ cells were injected subcutaneously in both sides of the mouse scapularis region and were allowed to grow for 3-4 weeks until they reached approximately $5 \mathrm{~mm}$ in diameter, as measured using a caliper. All experimental procedures were conducted under isoflurane gas anesthesia $(2-3 \%, 0.8 \mathrm{~L} / \mathrm{min})$. All animal experiments were performed following the treatment schedule shown in Figure 1. Briefly, pre-treatment images were obtained prior to irradiation to determine the size of the tumors based on bulk tumor GFP fluorescence. Tumor GFP fluorescence intensity is a delayed indicator of tumor response to irradiation since the GFP protein has a half-life of $\sim 26 \mathrm{~h}$ (42), ergo, GFP fluorescence intensity was not used to quantify tumor response following irradiation.

\section{Radiation Treatment and HQ4-DTPA Administration}

All irradiation procedures were performed using a small animal irradiation system (XRad 225Cx, Precision X-Ray Inc., North Branford, CT, USA) at a photon energy of $225 \mathrm{kVp}$ and a tube current of $13 \mathrm{~mA}$. Tumors were localized using x-ray fluoroscopy prior to irradiation. A $1.5 \mathrm{~cm}$ circular collimator was used to irradiate tumors at a dose rate of $2.9 \mathrm{~Gy} / \mathrm{min}$. The dose rate was measured using radiochromic films and a solid water phantom, as described previously (43). After delivery of the last radiation fraction, HQ4-DTPA was injected via the tail vein and anesthetized mice were imaged with each modality at 12,20 , and $40 \mathrm{~h}$ following injection (Figure 1).

\section{Fluorescence Imaging}

In vivo and ex vivo fluorescence images of GFP and HQ4-DTPA signals in MCF-7 tumors were obtained using the IVIS Spectrum imaging system (Perkin Elmer Inc., Waltham, MA, USA). GFP fluorescence signal was collected with an excitation wavelength of $465 \mathrm{~nm}$ and an emission wavelength of $500 \mathrm{~nm}$ ( $\pm 20 \mathrm{~nm})$. HQ4DTPA NIRF signal was collected with an excitation wavelength of $675 \mathrm{~nm}$ and an emission wavelength of $720 \mathrm{~nm}( \pm 20 \mathrm{~nm})$.

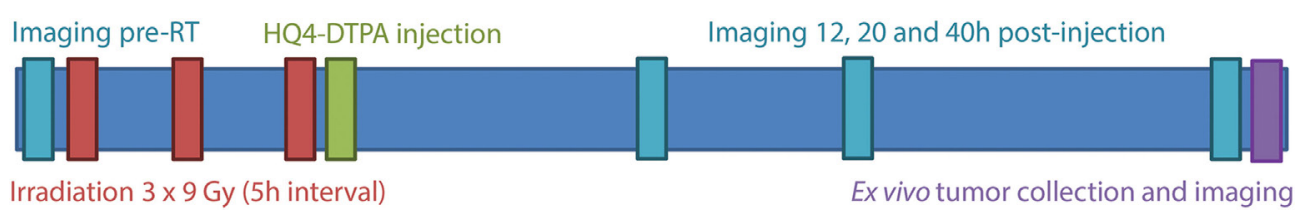

FIGURE 1 | Experimental schedule. Three fractions of 9 Gy irradiation were delivered with a 5-h interval and HQ4-DTPA was injected at the end of the irradiation schedule. In vivo photoacoustic and fluorescence imaging were performed at 12, 20, and $40 \mathrm{~h}$ following the injection. 


\section{Photoacoustic Imaging}

Tissue phantom, in vivo and ex vivo photoacoustic imaging of MCF-7 tumors was performed using the Vevo LAZR system (FujiFilm VisualSonics Inc., Toronto, ON, Canada) with a $21 \mathrm{MHz}$ center-frequency transducer. To prepare the phantom, HQ4-DTPA samples prepared at different concentrations (12.5, 25,50 , and $100 \mu \mathrm{M}$ ) were passed through polyethylene tubes that were placed on a piece of sliced turkey breast, having an approximate thickness of $2.5 \mathrm{~mm}$. Additional layers of meat were added to simulate various thicknesses of tissues. Photoacoustic images were obtained after the addition of each layer. For all experiments, 3D photoacoustic and ultrasound images were acquired simultaneously with a single wavelength of $700 \mathrm{~nm}$ for HQ4-DTPA, and the built-in Spectro mode was used to obtain the absorption spectrum from 680 to $900 \mathrm{~nm}$.

\section{SPECT-CT Imaging}

Mice were imaged at 12,20, and $40 \mathrm{~h}$ after intravenous injection of $\left.{ }^{111} \mathrm{In}\right] \mathrm{DTPA}-\mathrm{HQ} 4$. Mice were anesthetized by inhalation of $2 \%$ isoflurane in medical grade air. Imaging was performed on a nanoSPECT/CT system (Bioscan Inc., Washington, DC, USA) with four $\mathrm{NaI}(\mathrm{Tl})$ detectors fitted with $1.4-\mathrm{mm}$ multi-pinhole collimators (resolution $<1.2 \mathrm{~mm}$ at full-width-half-maximum). Cone beam CT images were acquired first (180 projections, $45 \mathrm{kVp}$ ), followed by the SPECT images. Photons were accepted from the $10 \%$ window centered on both the $245 \mathrm{keV}$ and $171 \mathrm{keV}$ photopeaks of ${ }^{111} \mathrm{In}$. A total of 24 projections were obtained in a $256 \times 256$ matrix for a total of $45 \mathrm{~min}$. The CT slices were reconstructed using a filtered back-projection algorithm, whereas the SPECT slices were reconstructed using an ordered subset expectation maximization (OSEM) algorithm with four subsets and nine iterations. CT and SPECT images were anatomically co-registered using the InVivoScope software (Bioscan/inviCRO, Boston, MA, USA).

Three mice were sacrificed after each experimental time point. Tissues were excised, weighed, and counted for radioactivity (PerkinElmer Wallac 1480 Wizard 3" gamma-counter, Waltham, MA, USA) along with a standard of the injected dose, so that the decay-corrected uptakes of HQ4-DTPA were determined as the percentage of the injected dose per gram (\% ID/g). The $\% \mathrm{ID} / \mathrm{g}$ was calculated as follows: [(MBq measured in tissue/injected dose $) \times 100 \% /$ weight of tissue]. The total injected dose per mouse was equal to the difference between the pre- and post-injection syringe radioactivity, as measured by a CRC-15R dose calibrator (Capintec, Ramsey, NJ, USA).

\section{Ex Vivo Fluorescence Imaging and Autoradiography}

Tumors were resected at the experimental endpoints, and were either embedded in OCT compound and snap frozen in liquid nitrogen, or fixed in formalin. Frozen sections were imaged using a phosphor imager (Cyclone Plus, Perkin Elmer) to detect ${ }^{111}$ In radioactivity. The same sections were subsequently imaged to measure HQ4-DTPA fluorescence with an excitation wavelength of $650 \mathrm{~nm}$ using TissueScope system (Huron Technologies). Formalin-fixed tissue sections were subjected to Hemotoxylin and Eosin (H\&E) staining and TdT-mediated dUTP Nick-End Labeling (TUNEL) staining (Promega, Madison, WI, USA) to detect radiation-induced tumor cell death including necrosis $(44,45)$.

\section{Statistical Analysis}

All statistical analyses were performed using GraphPad Prism ${ }^{\circledR}$ software (GraphPad Software, San Diego, CA, USA). Student's $t$-test was used to compare two sets of data, and two-way repeated measures ANOVA with Bonferroni post-test was used for serial imaging data. $p<0.05$ was considered significant, and error bars represent the mean \pm SEM.

\section{RESULTS}

\section{HQ4-DTPA as a Photoacoustic Contrast Agent}

To evaluate the application of HQ4-DTPA in addition to the NIRF property that was described previously (38), the photoacoustic property of carboxylated cyanine HQ4-DTPA was tested in a phantom composed of transparent plastic tubes. As seen in Figures 2A,B, HQ4-DTPA absorption increased with increasing concentration, demonstrating a peak at around $710 \mathrm{~nm}$ excitation. The photoacoustic absorption spectrum was similar to its known fluorescence spectrum (38), supporting its use as an extrinsic photoacoustic contrast agent.

To further characterize its performance as a photoacoustic contrast agent, multiple layers of meat were added over top of the tube phantoms to simulate a tissue thickness of up to $1 \mathrm{~cm}$. After the addition of each layer of meat, fluorescence and photoacoustic images, as well as photoacoustic absorption spectra, were acquired. In this way, we represented similar scattering and absorption patterns to those found in the human body. The fluorescence signals derived from the different concentrations were indistinguishable by the addition of the first layer of turkey breast tissue (2.5-mm thick) (data not shown). The photoacoustic intensity of the agent in the tubes was, however, detectable with layers up to $10 \mathrm{~mm}$ in total thickness at the highest concentration of HQ4-DTPA $(100 \mu \mathrm{M})$ (Figure 2C). Figure 2D shows the PA absorption spectra of $100 \mu \mathrm{M}$ HQ4-DTPA with the addition of 2.5-mm-thick tissue layers.

\section{In Vivo Serial Photoacoustic and Fluorescence Imaging of HQ4-DTPA Accumulation in Irradiated Tumors}

TdT-mediated dUTP nick-end labeling staining of irradiated tumor demonstrated over a two-fold difference in tumor cell death in tumors irradiated with three fractions of 9 Gy (27 Gy total), compared to non-irradiated control tumors (Figures 3A,B). H\&E staining was performed to confirm the TUNEL positive area as necrotic. The arrowheads in the image mark the difference in $\mathrm{H} \& \mathrm{E}$ staining between healthy and necrotic tissue. Based on those results, the same irradiation treatment regimen was used for all subsequent experiments. 

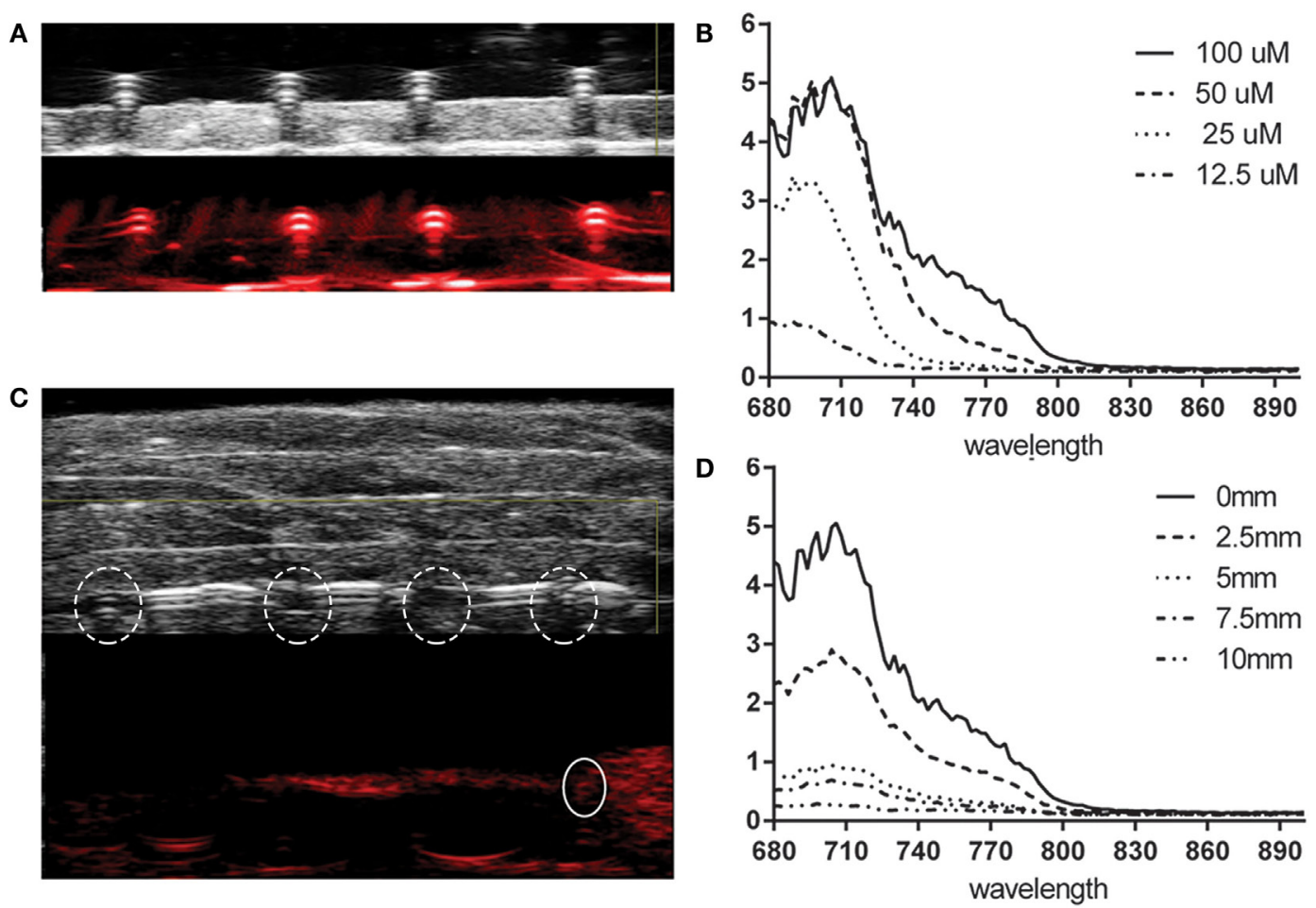

FIGURE 2 | Photoacoustic property of HQ4-DTPA. (A) Representative ultrasound (top) and photoacoustic (bottom) images of HQ4-DTPA in a tube phantom at different concentrations (from left to right: 12.5, 25, 50, and $100 \mu \mathrm{M}$ ). The photoacoustic image was acquired at $700 \mathrm{~nm}$. (B) Corresponding absorption spectra of HQ4-DTPA for the different concentrations. (C) Representative ultrasound (top) and photoacoustic (bottom) images of HQ4-DTPA in the same tube phantom as in (A), covered with 10-mm-thick meat. The dashed circles indicate the location of tubes, and the solid circle indicates the photoacoustic absorption of the tube containing $100 \mu \mathrm{M}$ HQ4-DTPA. (D) The corresponding absorption spectra of $100 \mu \mathrm{M}$ HQ4-DTPA with various thicknesses of meat covering the tube.
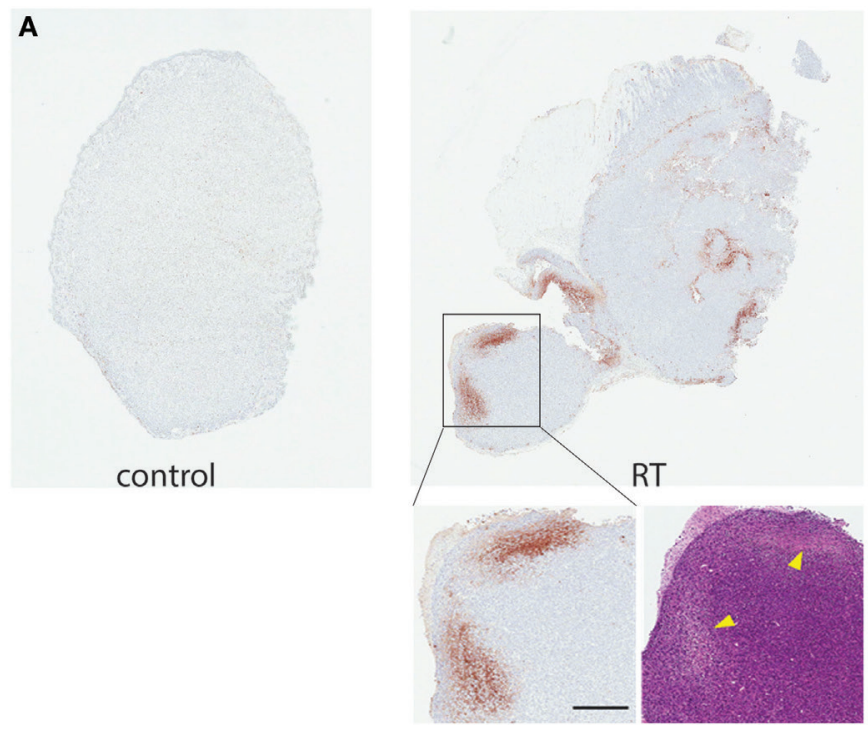

B

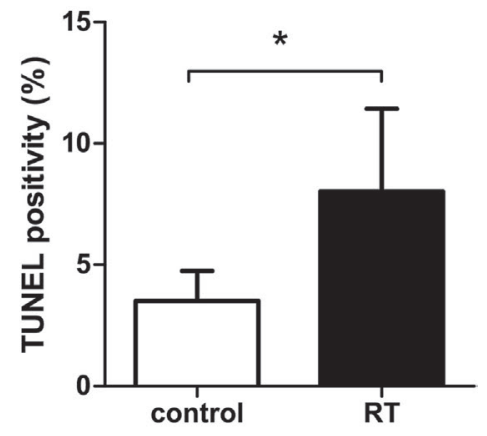

FIGURE 3 | Histological analysis of cell death after irradiation. (A) Representative images of TUNEL and H\&E staining for control and irradiated tumor resected $40 \mathrm{~h}$ after irradiation. The arrowheads point to necrotic areas. Scale bar $=500 \mu \mathrm{m}$. (B) Quantified TUNEL positivity for tumors resected $40 \mathrm{~h}$ after irradiation, expressed as \% positivity $\left(n=7 /\right.$ group, $\left.{ }^{*} p<0.05\right)$. 
As demonstrated in Figure $\mathbf{4 A}$, the photoacoustic images demonstrated accumulation of HQ4-DTPA inside the treated tumor mass, while some endogenous photoacoustic signals were observed in the outer rim of the tumor in both control and irradiated tumors. Figure 4B demonstrated a trend for increased accumulation of HQ4-DTPA in irradiated tumors compared to control tumors, although the difference was not statistically significant. The fluorescence images (Figures 4C,D) showed an approximate 1.8-fold increase in HQ4-DTPA accumulation in the irradiated subcutaneous tumors compared to non-irradiated controls, most notably at $12 \mathrm{~h}$ post-radiotherapy.

\section{Validation of Photoacoustic and Fluorescence Imaging of HQ4-DTPA in Ex Vivo Tissues}

To validate in vivo observations, control and irradiated tumors were resected $40 \mathrm{~h}$ following injection of HQ4-DTPA and subsequently imaged by photoacoustic and fluorescence systems. The resected masses were confirmed to be tumors based on the GFP fluorescence signal. As seen in Figure 5, increased HQ4-DTPA accumulation in an irradiated tumor was observed based on photoacoustic and fluorescence images. This indicated that the increased accumulation of HQ4-DTPA was specific to radiationinduced tumor cell necrosis in tumors. Since the skin covering the xenografted tumor was removed during resection, there was less interference from the intrinsic hemoglobin signal from blood vessels in the skin.

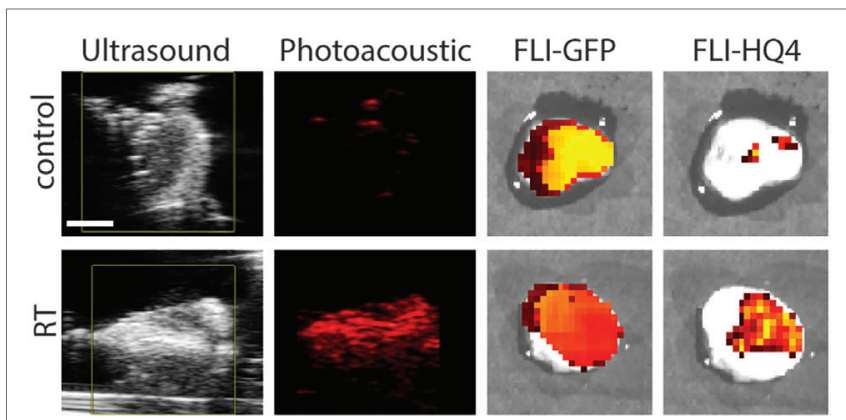

FIGURE 5 | Ex vivo photoacoustic and fluorescence imaging Representative ultrasound, photoacoustic, GFP fluorescence (FLI-GFP) and HQ4-DTPA fluorescence (FLI-HQ4) images of control and irradiated tumors resected $40 \mathrm{~h}$ after injection of $\mathrm{HQ} 4$. Scale bar $=2 \mathrm{~mm}$.

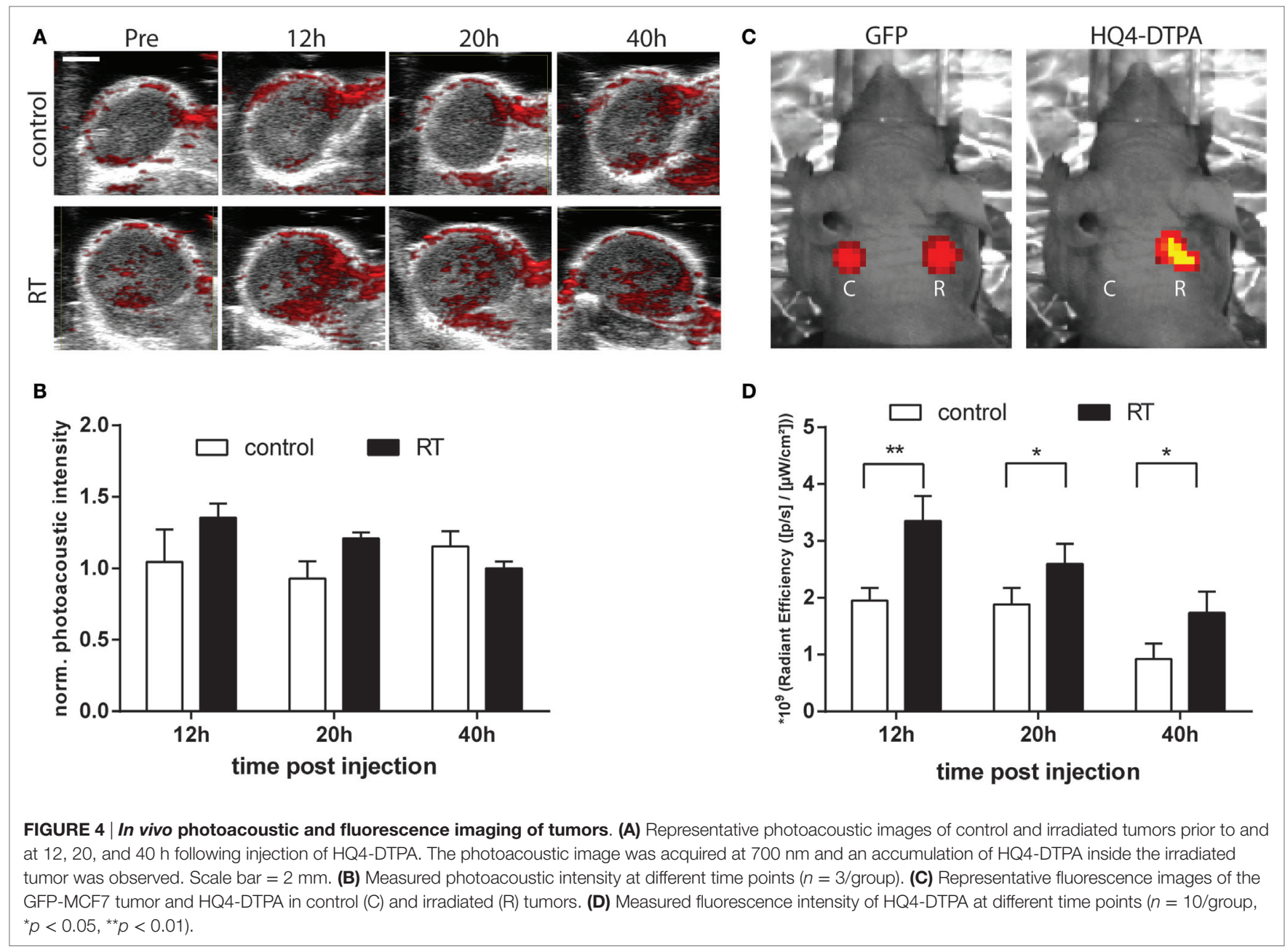




\section{In Vivo Biodistribution and Ex Vivo Validation of ${ }^{111}$ In Radiolabeled HQ4-DTPA}

SPECT-CT was performed to quantify whole-body biodistribution of [ ${ }^{111}$ In]DTPA-HQ4 in MCF-7 tumor-bearing mice following the experimental treatment schedule shown in Figure 1. Radiolabelling efficiency of HQ4-DTPA was determined to be greater than $90 \%$. Significantly higher accumulation of [ $\left.{ }^{111} \mathrm{In}\right] \mathrm{DTPA}-\mathrm{HQ} 4$ in irradiated tumors was observed compared to controls in the same mice $40 \mathrm{~h}$ after probe injection [tumorto-background ratio $\left.(\mathrm{TBR})=1.8, p_{40 \mathrm{~h}}=0.03\right]($ Figure 6A), thus confirming HQ4's specificity for necrotic tissues and suggesting the kinetics of HQ4-DTPA accumulation. Measurements of radioactivity in various resected organs demonstrated that $\left[{ }^{111} \mathrm{In}\right] \mathrm{DTPA}-\mathrm{HQ} 4$ was concentrated in the excreting organs with a peak in the kidneys, suggesting that the renal system was the main excreting route (Figure 6B). Lastly, the tumors were resected and imaged for [ ${ }^{111}$ In]DTPA-HQ4 using autoradiography and fluorescence. The autoradiographic images revealed a clear difference in structural characteristics between the irradiated and control tumors (Figures 7A,B). The internal tissue organization of the non-irradiated tumor was cohesive and showed a clear cellular pattern with a homogeneous color. By
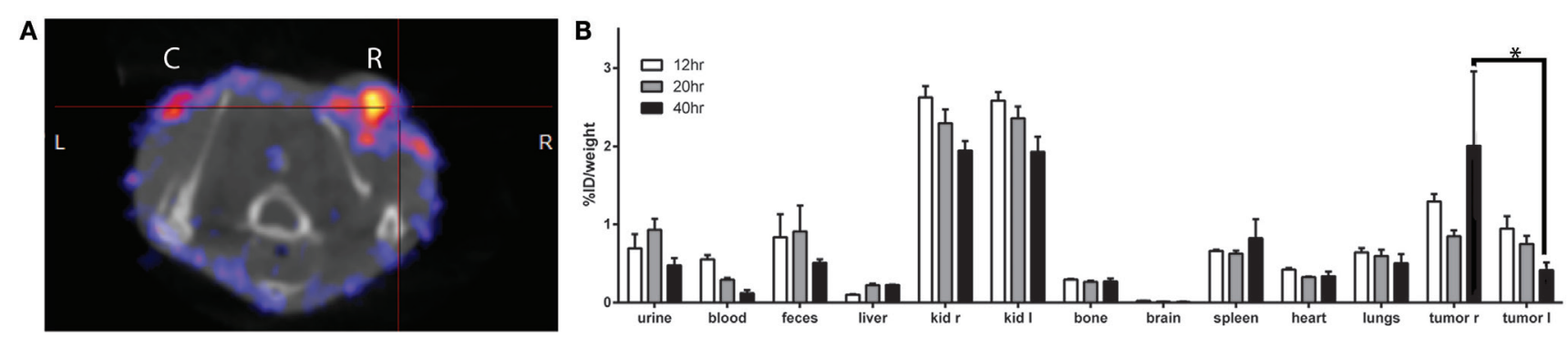

FIGURE 6 | SPECT-CT of [111In]DTPA-HQ4-DTPA biodistribution. (A) Representative transversal SPECT-CT image of the mouse with control (C) and irradiated (R) tumor $40 \mathrm{~h}$ following injection. (B) Measurement of [111In]DTPA-HQ4 biodistribution in\% ID/weight in different organs at various time points (12, 20, and $40 \mathrm{~h}$ post injection), where irradiated tumor ("tumor $r$ ") shows higher accumulation of the probe compared to control ("tumor l") $\left(n=3,{ }^{*} p<0.05\right)$.
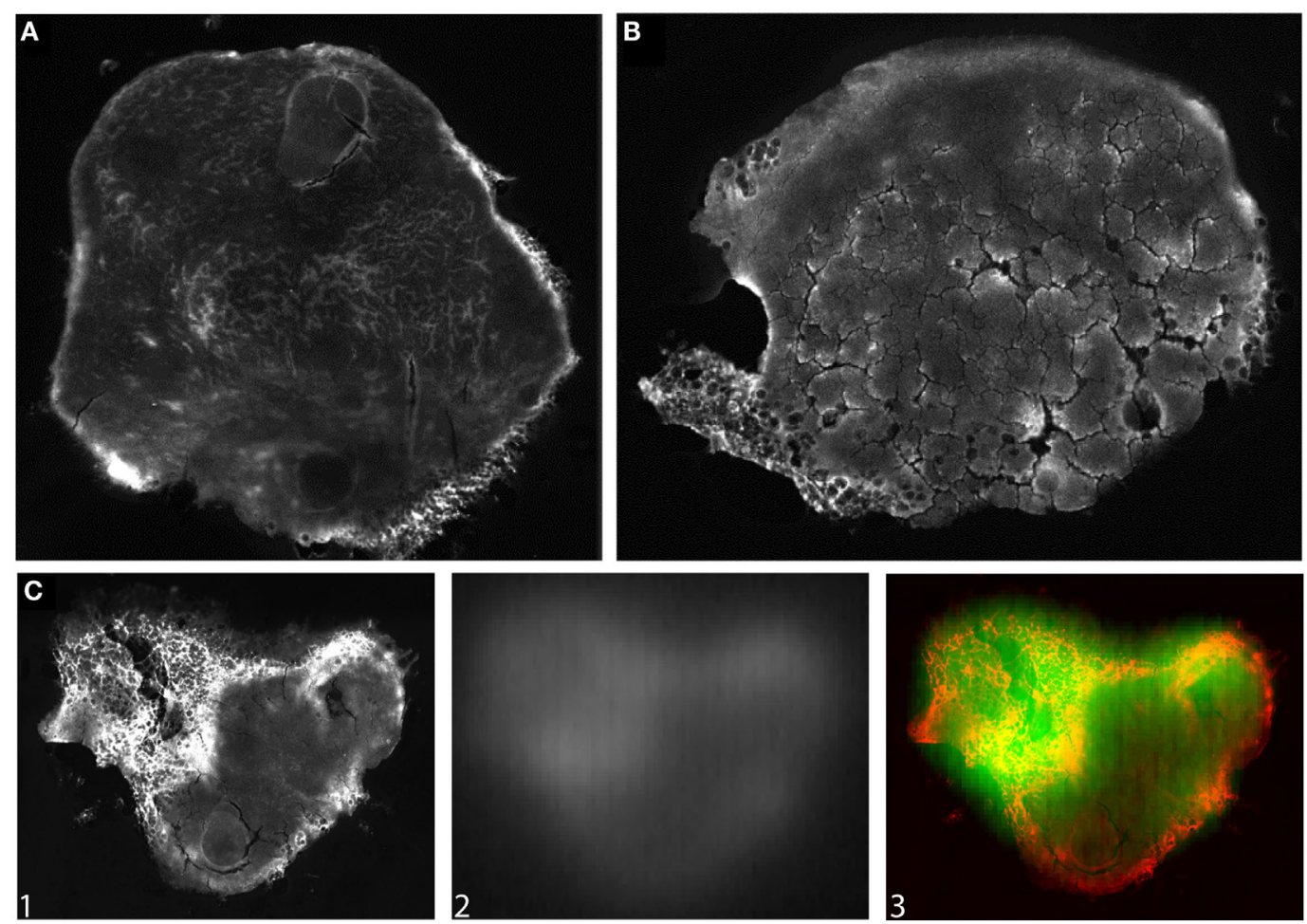

FIGURE 7 | Ex vivo fluorescence and autoradiography images of tumors. Representative images of (A) control and (B) irradiated tumor resected $40 \mathrm{~h}$ following injection of $\left[{ }^{111} \mathrm{I} n\right] \mathrm{DTPA}-\mathrm{HQ} 4$. The tumors were imaged for HQ4-DTPA fluorescence. (C) Overlay (C3) of HQ4-DTPA fluorescence (C1-red) and ${ }^{111}{ }^{1 n C l}{ }_{3}$ radioactivity (C2-green) in a tumor, illustrating co-localization of the two signals. 
contrast, the irradiated tumor showed a high level of disorganization. Furthermore, the overlay (C3) of fluorescence (C1-red) and autoradiography (C2-green) images showed a high degree of co-localization of ${ }^{111} \mathrm{In}_{-} \mathrm{Cl}_{3}$ and HQ4-DTPA fluorescence signal (Figure 7C).

\section{DISCUSSION}

In the current study, we evaluated HQ4-DTPA as a multimodal necrosis-avid imaging agent to assess tumor response to a clinically relevant radiotherapy dose using a MCF-7 human breast cancer mouse xenograft model. The necrosis-avid property of HQ4-DTPA for detection of chemotherapy-induced tumor cell necrosis was previously demonstrated using NIRF and SPECT (38). To extend the applicability of HQ4-DTPA to another imaging modality, we first assessed its photoacoustic property and demonstrated its distinct optical absorption peak at $\sim 700 \mathrm{~nm}$. Based on this result, multimodal imaging was performed to quantitatively evaluate the in vivo use of HQ4-DTPA to detect tumor response to radiotherapy using a fractionated irradiation scheme $(3 \times 9 \mathrm{~Gy})$. Our in vivo fluorescence results demonstrated an increase in HQ4-DTPA signal in irradiated tumors compared to non-irradiated tumors in vivo for up to $40 \mathrm{~h}$ after treatment, indicating specific and sustained accumulation of HQ4-DTPA in irradiated tumors. These data were supported by ex vivo NIRF and photoacoustic imaging of control and irradiated tumors. Lastly, we used SPECT-CT to quantitate the biodistribution of HQ4-DTPA, demonstrating HQ4-DTPA accumulation in irradiated tumors and clearance of unbound HQ4-DTPA mostly via kidneys, which was visualized at all time points. Collectively, our data indicated that HQ4-DTPA may be used as a multimodal necrosis-specific imaging agent. The data also suggested that HQ4-DTPA may be used clinically in the future to monitor solid tumor response to radiation therapy in a practical time frame.

Radiation therapy was selected as the treatment modality for the breast cancer model in the present study because of its widespread clinical usage. Breast conserving surgery is the standard treatment for localized breast cancer in combination with (neo-) adjuvant therapies (46), such as radiation therapy, which has been shown to reduce local recurrence (47). Radiation therapy is commonly administered in a conventional fractionated schedule ( 25 fractions of $2 \mathrm{~Gy}$ ) on the breast with an additional boost of up to 10 Gy on the lumpectomy cavity (47). Since such treatment schemes cannot be easily replicated in a relevant manner in animal models, we selected a radiation regimen that is isoeffective to a clinically relevant fractionated irradiation regimen based on a BED of $60 \mathrm{~Gy}$ in $2 \mathrm{~Gy}$ fractions $(48,49)$. Thus, the rationale for three fractions of 9 Gy with a 5-h interval was based on the BED for $60 \mathrm{~Gy}$, calculated using the $\alpha / \beta$ ratio of MCF-7 cells (4.62) $(50,51)$ and by taking into account the incomplete repair model based on the halftime of recovery from radiation damage in murine skin. Although the radiation regimen used in our study may not be used routinely or be clinically practical, we assumed that the regimen was appropriate to mimic the total radiation dose given in cancer patients as the BED is used to compare the relative effectiveness of different radiation protocols that vary in fraction size.
The NIRF properties of carboxylated cyanine HQ4-DTPA and its radioactive labeled variant $\left[{ }^{111} \mathrm{In}\right] \mathrm{DTPA}-\mathrm{HQ} 4$ have been previously demonstrated by our group (38). Although nuclear imaging overcomes the limited tissue penetration depth of NIRF imaging $(40,52)$, it has its drawbacks, including radiation safety, cost of radioactive materials, limited temporal sensitivity, and the lack of anatomical detail (53). Photoacoustic imaging overcomes such limitations and offers a novel and clinically relevant means of imaging HQ4-DTPA in vivo. Since photoacoustic imaging includes ultrasound imaging, both anatomical and functional information can be obtained simultaneously in real time. Photoacoustic imaging can image beyond the depth limitation of fluorescence imaging to more than $5 \mathrm{~cm}$ (54), making it suitable for imaging deeper tumors. In the current in vitro experiments, we distinguished a specific photoacoustic signal at a maximum depth of $1 \mathrm{~cm}$, achieving five times the tissue depth of NIRF imaging. However, depth imaging beyond $1 \mathrm{~cm}$ could not be performed due to the inherent property of the high-frequency ultrasound transducer $(21 \mathrm{MHz})$ used in our study. Photoacoustic imaging depth may be increased by using a lower frequency transducer, but at the expense of reduced detection sensitivity (55). Alternatively, a higher concentration of the probe may facilitate detection in deeper tissue.

Despite the advantages of photoacoustic imaging, there are some technical limitations. First, photoacoustic imaging may not be a suitable method for certain organs, such as lung and brain, where acoustic impedance is different between tissue interfaces (56). However, several preclinical studies demonstrated the use of photoacoustic imaging in these organs, suggesting future use of photoacoustic imaging in a variety of organs (57-59). Second, the clinical use of photoacoustic contrast must be approached cautiously, since photoacoustic imaging visualizes any tissue-based optical absorber at a given wavelength. As such, this method detects the presence of endogenous hemoglobin, a primary optical absorber in tissues, across a broad spectral range that includes 700-750 nm, corresponding to the peak absorption of HQ4. Our photoacoustic imaging results indicated the presence of an endogenous optical absorber mostly in the periphery of control and irradiated tumors, suggesting the presence of vasculature around the tumor. The endogenous absorption limited our ability to detect HQ4-DTPA in a highly specific manner. To distinguish absorption by any contrast agent from that of endogenous absorbers, photoacoustic spectral unmixing techniques can be performed to obtain a clear overview of the contrast agent signal based on its known spectrum (60). Such techniques can be applied in future studies to visualize the accumulation of HQ4-DTPA inside the irradiated tumors in a specific manner. In addition, the imaging probe may accumulate inside tumors due to intrinsic tumor necrosis resulting in the presence of background signal in both fluorescence and photoacoustic imaging. In such cases, baseline imaging needs to be performed with the injection of HQ4-DTPA prior to initiation of an anticancer treatment.

Although NIRF imaging is widely clinically applicable, its use as a singular imaging modality to assess biological activities may be suboptimal. For example, fluorescence properties of exogenous dyes used in vivo are strongly influenced by the tissue 
microenvironment, such as hydrophobicity and $\mathrm{pH}$, as well as by interactions with various proteins $(61,62)$. Such interactions will influence HQ4-DTPA fluorescence intensity differently in an in vivo environment of living cells, which may hamper quantification of probe accumulation inside necrotic tumors. These same interactions may have contributed to the differences in the time point of highest signal accumulation observed using the different imaging methods in our model, although it was not explicitly addressed in this study. To achieve absolute quantification of a probe, gamma spectroscopy or mass spectrometry should be considered $(61,62)$. In our study, quantification of HQ4-DTPA was achieved by measurement of radioactivity in various organs, supporting the in vivo imaging data in a quantitative manner.

Irradiation causes direct DNA damage and the production of reactive oxygen species (ROS), both leading to cell death. The amount and the type of cell death depend on the tumor type and the irradiation dose per fraction. For MCF-7 cells, the $\alpha / \beta$ ratio, a model of radiation effect, is relatively low compared to the higher $\alpha / \beta$ ratios for other tumors, such as Tara- $1 / 2$ (teratoma), DU145 (bladder), TSU, and UNCap (prostate) (e.g., 7-20 Gy) (51), suggesting that the treatment response may be delayed in MCF-7 tumor-bearing animals. This delayed response can be seen by our in vivo radioactivity-based biodistribution results demonstrating significant differences of HQ4-DTPA accumulation between the treated and control tumor $40 \mathrm{~h}$ after irradiation. This discrepancy may increase even more over time, requiring re-injection of the probe at a later time point or at multiple time points following radiotherapy. In addition, radiation-induced damage may be more severe when high dose of radiation is used per fraction, leading to direct tumor cell destruction as well as secondary tumor cell death $(63,64)$. Therefore, future studies may focus on multifractionated scheme with a lower fraction dose to assess whether the proposed necrosis-imaging technique is still applicable. In testing multi-fractionation schemes, the imaging technique could be initially tested in the same way so immediately after the end of the complete treatment, and later on even during the treatment process to assess its utility in adapting therapeutic regimen. In the current study, we chose to inject HQ4-DTPA immediately after the final tumor irradiation to detect early treatment response since the goal of this study was to investigate HQ4-DTPA imaging as an early indicator of radiation-induced necrosis, Collectively, future studies are warranted with multi-fractionation scheme and/or injections at multiple time points to evaluate its utility in treatment monitoring and adaptive treatment.

Overall, we have demonstrated that HQ4-DTPA can be used to objectively assess tumor response to radiation therapy.

\section{REFERENCES}

1. Ferlay J, Soerjomataram I, Dikshit R, Eser S, Mathers C, Rebelo M, et al. Cancer incidence and mortality worldwide: sources, methods and major patterns in GLOBOCAN 2012. Int J Cancer (2015) 136:E359-86. doi:10.1002/ijc.29210

2. Eisenhauer EA, Therasse P, Bogaerts J, Schwartz LH, Sargent D, Ford R, et al. New response evaluation criteria in solid tumours: revised RECIST guideline (version 1.1). Eur J Cancer (2009) 45:228-47. doi:10.1016/j.ejca.2008.10.026

3. Fournier L, Ammari S, Thiam R, Cuénod CA. Imaging criteria for assessing tumour response: RECIST, mRECIST, Cheson. Diagn Interv Imaging (2014) 95:689-703. doi:10.1016/j.diii.2014.05.002
HQ4-DTPA is distinct from current clinically available necrosisavid agents given its unique in vivo specificity and multimodal imaging capability. The added benefit of multimodal imaging potentially broadens its applicability in a variety of clinical settings, where tissue necrosis serves as a surrogate marker of diseases as well as response to necrosis-inducing treatments. The advantages of the small molecule [ ${ }^{111}$ In]DTPA-HQ4 include high water solubility, the photoacoustic property that enables deep tissue penetration into tissues, lack of phototoxicity, and low production costs. Unlike fluorescence imaging and SPECT, photoacoustic imaging combines the anatomical and functional properties of tissue in a 3D image. Therefore, the necrosis avid radiotracer $\left[{ }^{111} \mathrm{In}\right] \mathrm{DTPA}-\mathrm{HQ} 4$ has the potential to be clinically translated for diagnostic and prognostic purposes, as well as to predict early treatment outcome of antitumor treatments, such as radiation therapy. Additional preclinical and clinical studies are required to demonstrate the advantages of this novel imaging approach to assess early treatment efficacy and inform adaptive therapy decisions for individual patients.

\section{AUTHOR CONTRIBUTIONS}

The author contribution can be divided into several parts in which several authors played an essential role. Those parts and the contributions are described below: Conception of the work: MS, AM, DS, PM, ED, AC, CL, and RD. Design of the work: MS, $\mathrm{AM}, \mathrm{JB}, \mathrm{DS}, \mathrm{RS}, \mathrm{TS}$, and ER. Acquisition of the work: MS, AM, JB, DS, and RS. Analysis/interpretation of the work: MS, AM, PM, TS, ER, AC, CL, and RD. Drafting the work: MS, AM, IK, and RD. Revising the work: MS, AM, DS, IK, ED, CL, and RD. Final approval: MS, AM, JB, DS, IK, PM, RS, ED, TS, EB, AC, CL, and RD. Agreement to be accountable: MS, AM, JB, DS, IK, PM, RS, $\mathrm{ED}, \mathrm{TS}, \mathrm{EB}, \mathrm{AC}, \mathrm{CL}$, and RD.

\section{FUNDING}

This work was supported by funding provided to RD by the Canadian Institutes of Health Research, Terry Fox Research Institute and by the International Collaboration R\&D Program, South Korean Ministry of Knowledge Economy. The authors would like to acknowledge the Spatio-Temporal Targeting and Amplification of Radiation Response (STTARR) program and its affiliated funding agencies. Additionally, this work was supported by project grants from the EU Programme: FP7-PEOPLE2013-IAPP (612360 - BRAINPATH) and H2020-MSCA-RISE (644373 - PRISAR).

4. Han Z, Fu A, Wang H, Diaz R, Geng L, Onishko H, et al. Noninvasive assessment of cancer response to therapy. Nat Med (2008) 14:343-9. doi:10.1038/ nm1691

5. Nishino M, Jagannathan JP, Krajewski KM, O’Regan K, Hatabu H, Shapiro G, et al. Personalized tumor response assessment in the era of molecular medicine: cancer-specific and therapy-specific response criteria to complement pitfalls of RECIST. AJR Am J Roentgenol (2012) 198:737-45. doi:10.2214/ AJR.11.7483

6. Zhao B, Schwartz LH, Larson SM. Imaging surrogates of tumor response to therapy: anatomic and functional biomarkers. J Nucl Med (2009) 50:239-49. doi:10.2967/jnumed.108.056655 
7. Silva MT. Secondary necrosis: the natural outcome of the complete apoptotic program. FEBS Lett (2010) 584:4491-9. doi:10.1016/j.febslet.2010.10.046

8. Uhl M, Saueressig U, Koehler G, Kontny U, Niemeyer C, Reichardt W, et al. Evaluation of tumour necrosis during chemotherapy with diffusion-weighted MR imaging: preliminary results in osteosarcomas. Pediatr Radiol (2006) 36:1306-11. doi:10.1007/s00247-006-0324-x

9. Eriksson D, Stigbrand T. Radiation-induced cell death mechanisms. Tumor Biol (2010) 31:363-72. doi:10.1007/s13277-010-0042-8

10. Fink SL, Cookson BT. Apoptosis, pyroptosis, and necrosis: mechanistic description of dead and dying eukaryotic cells. Infect Immun (2005) 73:1907-16. doi:10.1128/IAI.73.4.1907-1916.2005

11. Nikoletopoulou V, Markaki M, Palikaras K, Tavernarakis N. Crosstalk between apoptosis, necrosis and autophagy. Biochim Biophys Acta (2013) 1833:3448-59. doi:10.1016/j.bbamcr.2013.06.001

12. Herman AB, Savage VM, West GB. A quantitative theory of solid tumor growth, metabolic rate and vascularization. PLoS One (2011) 6:e22973. doi:10.1371/journal.pone. 0022973

13. Hiraoka N, Ino Y, Sekine S, Tsuda H, Shimada K, Kosuge T, et al. Tumour necrosis is a postoperative prognostic marker for pancreatic cancer patients with a high interobserver reproducibility in histological evaluation. $\mathrm{Br}$ J Cancer (2010) 103:1057-65. doi:10.1038/sj.bjc.6605854

14. Kato T, Kameoka S, Kimura T, Tanaka S, Nishikawa T, Kobayashi M. p53, mitosis, apoptosis and necrosis as prognostic indicators of long-term survival in breast cancer. Anticancer Res (2002) 22:1105-12.

15. Maiorano E, Regan MM, Viale G, Mastropasqua MG, Colleoni M, CastiglioneGertsch M, et al. Prognostic and predictive impact of central necrosis and fibrosis in early breast cancer: results from two International Breast Cancer Study Group randomized trials of chemoendocrine adjuvant therapy. Breast Cancer Res Treat (2010) 121:211-8. doi:10.1007/s10549-009-0360-y

16. Park SY, Lee HS, Jang HJ, Lee GK, Chung KY, Zo JI. Tumor necrosis as a prognostic factor for stage IA non-small cell lung cancer. Ann Thorac Surg (2011) 91:1668-73. doi:10.1016/j.athoracsur.2010.12.028

17. Pichler M, Hutterer GC, Chromecki TF, Jesche J, Kampel-Kettner K, Rehak $\mathrm{P}$, et al. Histologic tumor necrosis is an independent prognostic indicator for clear cell and papillary renal cell carcinoma. Am J Clin Pathol (2012) 137:283-9. doi:10.1309/AJCPLBK9L9KDYQZP

18. Pollheimer MJ, Kornprat P, Lindtner RA, Harbaum L, Schlemmer A, Rehak P, et al. Tumor necrosis is a new promising prognostic factor in colorectal cancer. Hum Pathol (2010) 41:1749-57. doi:10.1016/j.humpath.2010.04.018

19. Kepp O, Galluzzi L, Lipinski M, Yuan J, Kroemer G. Cell death assays for drug discovery. Nat Rev Drug Discov (2011) 10:221-37. doi:10.1038/nrd3373

20. Prinsen K, Jin L, Vunckx K, De Saint-Hubert M, Zhou L, Cleynhens J, et al. Radiolabeling and preliminary biological evaluation of a $(99 \mathrm{~m}) \mathrm{Tc}(\mathrm{CO})(3)$ labeled 3,3'-(benzylidene)-bis-(1H-indole-2-carbohydrazide) derivative as a potential SPECT tracer for in vivo visualization of necrosis. Bioorg Med Chem Lett (2011) 21:502-5. doi:10.1016/j.bmcl.2010.10.102

21. Venkatramani R, Wang L, Malvar J, Dias D, Sposto R, Malogolowkin MH, et al. Tumor necrosis predicts survival following neo-adjuvant chemotherapy for hepatoblastoma. Pediatr Blood Cancer (2012) 59:493-8. doi:10.1002/ pbc. 24038

22. Van Walleghen DM, Parseghian MH. Toxicity and biodistribution of an iodine-131-radiolabelled tumour necrosis-targeting antibody in non-tumour-bearing domestic felines. Vet Comp Oncol (2006) 4:9-20. doi:10.1111/ j.1476-5810.2006.00086.x

23. Richards CH, Roxburgh CS, Anderson JH, McKee RF, Foulis AK, Horgan PG, et al. Prognostic value of tumour necrosis and host inflammatory responses in colorectal cancer. Br J Surg (2012) 99:287-94. doi:10.1002/bjs.7755

24. Epstein AL, Chen FM, Taylor CR. A novel method for the detection of necrotic lesions in human cancers. Cancer Res (1988) 48:5842-8.

25. Jiang B, Wang J, Ni Y, Chen F. Necrosis avidity: a newly discovered feature of hypericin and its preclinical applications in necrosis imaging. Theranostics (2013) 3:667-76. doi:10.7150/thno.6650

26. Xie B, Stammes MA, van Driel PB, Cruz LJ, Knol-Blankevoort VT, Lowik $\mathrm{MA}$, et al. Necrosis avid near infrared fluorescent cyanines for imaging cell death and their use to monitor therapeutic efficacy in mouse tumor models. Oncotarget (2015) 6(36):39036-49. doi:10.18632/oncotarget.5498

27. Verma N, Cowperthwaite MC, Burnett MG, Markey MK. Differentiating tumor recurrence from treatment necrosis: a review of neuro-oncologic imaging strategies. Neuro Oncol (2013) 15:515-34. doi:10.1093/neuonc/nos307
28. Murphy KP, O'Connor OJ, Maher MM. Updated imaging nomenclature for acute pancreatitis. AJR Am J Roentgenol (2014) 203:W464-9. doi:10.2214/ AJR.13.12222

29. Carlsson M, Arheden H, Higgins CB, Saeed M. Magnetic resonance imaging as a potential gold standard for infarct quantification. J Electrocardiol (2008) 41:614-20. doi:10.1016/j.jelectrocard.2008.06.010

30. Cona MM, de Witte P, Verbruggen A, Ni Y. An overview of translational (radio) pharmaceutical research related to certain oncological and non-oncological applications. World J Methodol (2013) 3:45-64. doi:10.5662/wjm.v3.i4.45

31. Ni Y. Abstract 1767: oncocidia: a small molecule dual targeting pan-anticancer theragnostic strategy. Paper Presented at: Proceedings of the 105th Annual Meeting of the American Association for Cancer Research. San Diego, CA; Philadelphia, PA (2014).

32. Cona MM, Li J, Feng Y, Chen F, Verbruggen A, Witte P, et al. Targetability and biodistribution of radioiodinated hypericin: comparison between microdosing and carrier-added preparations. Anticancer Agents Med Chem (2014) 14:852-61. doi:10.2174/18715206113136660360

33. Hdeib A, Sloan A. Targeted radioimmunotherapy: the role of (1)(3)(1) I-chTNT-1/B mAb (Cotara) for treatment of high-grade gliomas. Future Oncol (2012) 8:659-69. doi:10.2217/fon.12.58

34. Pharmaceuticals P. Cotara Oncology. (2015). Available from: http://www. peregrineinc.com/pipeline/cotara-oncology.html

35. Hritz J, Kascakova S, Ulicny J, Miskovsky P. Influence of structure of human, rat, and bovine serum albumins on binding properties of photoactive drug hypericin. Biopolymers (2002) 67:251-4. doi:10.1002/bip.10110

36. Solar P, Cavarga I, Hofmanova J, Cekanova-Figurova M, Miskovsky P, Brezani P, et al. Effect of acetazolamide on hypericin photocytotoxicity. Planta Med (2002) 68:658-60. doi:10.1055/s-2002-32902

37. Wang H, Cao C, Li B, Chen S, Yin J, Shi J, et al. Immunogenicity of Iodine 131 chimeric tumor necrosis therapy monoclonal antibody in advanced lung cancer patients. Cancer Immunol Immunother (2008) 57:677-84. doi:10.1007/ s00262-007-0406-0

38. Stammes MA, Knol-Blankevoort VT, Cruz LJ, Feitsma HR, Mezzanotte L, Cordfunke RA, et al. Pre-clinical evaluation of a cyanine-based SPECT probe for multimodal tumor necrosis imaging. Mol Imaging Biol (2016). doi:10.1007/ s11307-016-0972-7

39. Delaney G, Jacob S, Featherstone C, Barton M. The role of radiotherapy in cancer treatment. Cancer (2005) 104:1129-37. doi:10.1002/cncr.21324

40. Smith AM, Mancini MC, Nie S. Second window for in vivo imaging. Nat Nanotechnol (2009) 4:710-1. doi:10.1038/nnano.2009.326

41. Brom M, Joosten L, Oyen WJ, Gotthardt M, Boerman OC. Improved labelling of DTPA- and DOTA-conjugated peptides and antibodies with 111In in HEPES and MES buffer. EJNMMI Res (2012) 2:4. doi:10.1186/2191219X-2-4

42. Corish P, Tyler-Smith C. Attenuation of green fluorescent protein halflife in mammalian cells. Protein Eng (1999) 12:1035-40. doi:10.1093/ protein/12.12.1035

43. Stewart JMP, Lindsay PE, Jaffray DA. Two-dimensional inverse planning and delivery with a preclinical image guided microirradiator. Med Phys (2013) 40:101709. doi:10.1118/1.4819935

44. de Torres C, Munell F, Ferrer I, Reventós J, Macaya A. Identification of necrotic cell death by the TUNEL assay in the hypoxic-ischemic neonatal rat brain. Neurosci Lett (1997) 230:1-4. doi:10.1016/S0304-3940(97)00445-X

45. Loo DT. Situ detection of apoptosis by the TUNEL assay: an overview of techniques. In: Didenko VV, editor. DNA Damage Detection In Situ, Ex Vivo, and In Vivo: Methods and Protocols. Totowa, NJ: Humana Press (2011). p. 3-13.

46. Nounou MI, ElAmrawy F, Ahmed N, Abdelraouf K, Goda S, Syed-Sha-Qhattal $\mathrm{H}$. Breast cancer: conventional diagnosis and treatment modalities and recent patents and technologies. Breast Cancer (Auckl) (2015) 9:17-34. doi:10.4137/ BCBCR.S29420

47. Lee E, Takita C, Wright JL, Reis IM, Zhao W, Nelson OL, et al. Characterization of risk factors for adjuvant radiotherapy-associated pain in a tri-racial/ ethnic breast cancer population. Pain (2016) 157:1122-31. doi:10.1097/j. pain. 0000000000000489

48. Kyrgias G, Zygogianni A, Theodorou K, Koukourakis M, Oikonomou A, Kouvaris J, et al. Accelerated hypofractionated whole-breast irradiation with concomitant daily boost in early breast cancer. Am J Clin Oncol (2015) 38:358-63. doi:10.1097/COC.0b013e3182a46740 
49. Zygogianni A, Kouloulias V, Antypas C, Armpilia C, Kyrgias G, Kouvaris J. The impact of intermediate time between chemotherapy and hypofractionated radiotherapy to the radiation induced skin toxicity for breast adjuvant treatment. Breast J (2014) 20:74-8. doi:10.1111/tbj.12206

50. Williams JR, Zhang Y, Zhou H, Gridley DS, Koch CJ, Russell J, et al. A quantitative overview of radiosensitivity of human tumor cells across histological type and TP53 status. Int J Radiat Biol (2008) 84:253-64. doi:10.1080/09553000801953342

51. Joiner M, van der Kogel A. Basic Clinical Radiobiology. London: Hodder Arnold (2009).

52. Xie B, Stammes MA, van Driel PBAA, Cruz LJ, Knol-Blankevoort VT, Löwik MAM, et al. Necrosis avid near infrared fluorescent cyanines for imaging cell death and their use to monitor therapeutic efficacy in mouse tumor models. Oncotarget (2015) 6:39036-49. doi:10.18632/oncotarget.5498

53. Erdi YE. Limits of tumor detectability in nuclear medicine and PET. Mol Imag Radionucl Ther (2012) 21:23-8. doi:10.4274/Mirt.138

54. Kim C, Erpelding TN, Jankovic L, Pashley MD, Wang LV. Deeply penetrating in vivo photoacoustic imaging using a clinical ultrasound array system. Biomed Opt Express (2010) 1:278-84. doi:10.1364/BOE.1.000278

55. Yao J, Wang LV. Photoacoustic microscopy. Laser Photonics Rev (2013) 7(5):758-78. doi:10.1002/lpor.201200060

56. Beard P. Biomedical photoacoustic imaging. Interface Focus (2011) 1:602-31. doi:10.1098/rsfs.2011.0028

57. Raes F, Sobilo J, Le Mée M, Rétif S, Natkunarajah S, Lerondel S, et al. High resolution ultrasound and photoacoustic imaging of orthotopic lung cancer in mice: new perspectives for onco-pharmacology. PLoS One (2016) 11:e0153532. doi:10.1371/journal.pone.0153532

58. Wang D, Wu Y, Xia J. Review on photoacoustic imaging of the brain using nanoprobes. Neurophotonics (2016) 3:010901. doi:10.1117/1.NPh.3.1.010901
59. Yao J, Wang LV. Photoacoustic brain imaging: from microscopic to macroscopic scales. Neurophotonics (2014) 1:011003. doi:10.1117/1.NPh.1.1.011003

60. Luke GP, Nam SY, Emelianov SY. Optical wavelength selection for improved spectroscopic photoacoustic imaging. Photoacoustics (2013) 1:36-42. doi:10.1016/j.pacs.2013.08.001

61. Liu Y, Tseng Y-C, Huang L. Biodistribution studies of nanoparticles using fluorescence imaging: a qualitative or quantitative method? Pharm Res (2012) 29:3273-7. doi:10.1007/s11095-012-0818-1

62. Hawe A, Sutter M, Jiskoot W. Extrinsic fluorescent dyes as tools for protein characterization. Pharm Res (2008) 25:1487-99. doi:10.1007/s11095-007-9516-9

63. Hellevik T, Martinez-Zubiaurre I. Radiotherapy and the tumor stroma: the importance of dose and fractionation. Front Oncol (2014) 4:1. doi:10.3389/ fonc. 2014.00001

64. Song CW, Cho LC, Yuan J, Dusenbery KE, Griffin RJ, Levitt SH. Radiobiology of stereotactic body radiation therapy/stereotactic radiosurgery and the linear-quadratic model. Int J Radiat Oncol Biol Phys (2013) 87:18-9. doi:10.1016/j.ijrobp.2013.03.013

Conflict of Interest Statement: $\mathrm{HQ}^{\mathrm{TM}}$ compounds are a trade mark of Ilumicare BV, Rotterdam. The authors have no other conflicts of interest to declare.

Copyright (c) 2016 Stammes, Maeda, Bu, Scollard, Kulbatski, Medeiros, Sinisi, Dubikovskaya, Snoeks, van Beek, Chan, Löwik and DaCosta. This is an open-access article distributed under the terms of the Creative Commons Attribution License (CC $B Y)$. The use, distribution or reproduction in other forums is permitted, provided the original author(s) or licensor are credited and that the original publication in this journal is cited, in accordance with accepted academic practice. No use, distribution or reproduction is permitted which does not comply with these terms. 